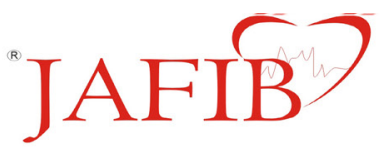

Featured Review

\title{
Journal of Atrial Fibrillation
}

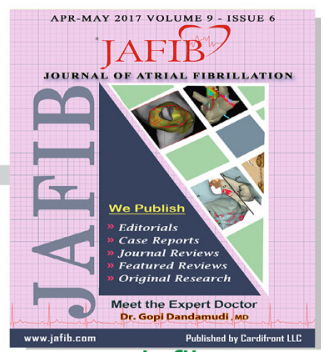

www. jafib.com

\section{Incorporating Stroke and Bleeding Risk Stratification Tools Into Atrial Fibrillation Management Making Sense of the Alphabet Soup}

Thomas F Deering

Arrhythmia Center: Piedmont Heart Institute.

\section{Introduction}

Atrial fibrillation (AF) is a very common rhythm disturbance, which is increasing in both incidence and prevalence globally. Some estimates predict a doubling in the incidence of and mortality rates related to $\mathrm{AF}$ over the next thirty years. ${ }^{[1],[2]}$ These demographic and outcome projections demonstrate the need for the medical community to continue developing effective treatments for AF and the need of clinicians and society to pursue policies that will minimize the negative clinical consequences associated with AF.

Patients with AF often have an impaired quality of life due to a number of associated problematic arrhythmic issues such as tachycardia, bradycardia, loss of AV synchrony and rate irregularity. $\mathrm{AF}$ patients are also at higher risk for more deleterious clinical outcomes such as a tachycardia-mediated cardiomyopathy, stroke and death. ${ }^{[3]}$ Cardio-embolic and cryptogenic strokes, many of which are secondary to thrombogenic processes associated with $\mathrm{AF}$, together account for almost $50 \%$ of observed strokes ${ }^{[4]}$ Furthermore, when strokes occur in the setting of AF, they are often more disabling and associated with a higher mortality rate than strokes deriving from other etiologies. ${ }^{[5]} \mathrm{AF}$-related stokes also have significant economic implications. Patients sustaining a stroke in the setting of AF have higher overall index and longitudinal medical costs than do patients with strokes resulting from other causes. ${ }^{[6]}$

Accordingly, preventing stroke is one of the most important tasks with which the medical community rendering care to $\mathrm{AF}$ patients is charged. In this review the importance of performing a thromboembolic and bleeding risk stratification assessment among all $\mathrm{AF}$ patients to determine the therapeutic risk: benefit ratios for oral anticoagulant $(\mathrm{OAC})$ therapy will be discussed. This analysis will be followed by a review about how this algorithmic thromboembolic and bleeding risk stratification information can be used directly to determine the proper pharmacologic approach designed to lower stroke rates and minimize bleeding complications among divergent AF patient populations. The manuscript will conclude with a review of the status of global initiatives to create well-developed oral Key Words Atrial Fibrillation, Stroke, Risk Stratification.

Corresponding Author

Thomas F. Deering, MD

(thomas.deering@piedmont.org) anticoagulation programs.

Early Oral Anticoagulant Development And Algorithmic Usage In Atrial Fibrillation Patients

Since the isolation of warfarin by Campbell and Paul in the first half of the twentieth century OAC therapy has evolved to become a cornerstone in the treatment of patients with AF to reduce the risk of thromboembolic events, especially stroke. ${ }^{[7]}$ In a number of pivotal trials warfarin was shown to be substantially better than placebo in lowering both stroke rates and mortality rates. In a meta-analysis of these older landmark trials Hart demonstrated an overall $62 \%$ relative stroke risk reduction and a $26 \%$ relative all-cause mortality risk reduction. ${ }^{[8]}$

However, the risk for thromboembolic events is quite variable among AF patients. Accordingly, a number of algorithms have been developed in an attempt to clarify more effectively the stroke risk so that the clinical decision-making processes can be tailored to align with individual patient profiles. The two most commonly employed algorithms to define the thromboembolic risk are the $\mathrm{CHADS}_{2}$ and $\mathrm{CHA}_{2} \mathrm{DS}_{2}$-VASc algorithms [Table 1]. ${ }^{[9],[10]}$ Although widely used and clinically valuable, each of these algorithms has a number of limitations. Both were developed among focused patient populations with limited information about subsequent anticoagulation usage, minimal information about the post-discharge methods employed to define clinical outcomes and no original independent external validation.

The $\mathrm{CHADS}_{2}$ And $\mathrm{CHA}_{2} \mathrm{DS}_{2}$-Vasc Algorithms: Defining The Atrial Fibrillation Patient At Low Risk For A Thromboembolic Event

Although there is overlap in the ability of the two algorithms to define thromboembolic risk, the $\mathrm{CHA}_{2} \mathrm{DS}_{2}-\mathrm{VASc}$ algorithm includes more demographic and clinical components to characterize that risk. By including more risk factors into the scoring algorithm for the $\mathrm{CHA}_{2} \mathrm{DS}_{2}$-VASc score, its developers hoped that it would result in a more accurate risk stratification process by categorizing more effectively patients into low, medium or high risk sub-groups than was possible with the CHADS 2 algorithm.

The initial risk stratification requirement, with which clinicians are faced, is the need to correctly define those patients, who are at low risk for a stroke. By accurately characterizing this group one 
can identify patients in whom it would be prudent clinically to withhold OAC therapy. To this end, the available data suggest that the overall ability of the $\mathrm{CHADS}_{2}$ score to identify low stroke risk patients is very limited, based on a number of published studies in which a variable stroke rate, ranging from $0.9 \%$ to $2.8 \%$, has been reported among patients with a $\mathrm{CHADS}_{2}$ score of 0 [Figure 1]. [11]-[15] The ability of the $\mathrm{CHADS}_{2}$ and $\mathrm{CHA}_{2} \mathrm{DS}_{2}-\mathrm{VASc}$ scores to predict stroke rates among potentially low risk patients was compared in a

\begin{tabular}{|c|c|c|c|}
\hline \multirow{2}{*}{$\begin{array}{l}\text { Table 1: } \begin{array}{l}\text { Stro } \\
\text { Scor }\end{array} \\
\text { Risk Factor }\end{array}$} & $\begin{array}{l}\text { Risk Stratifica } \\
\text { Algorithms }\end{array}$ & \multirow[b]{2}{*}{ Risk Factor } & \multirow[b]{2}{*}{$\mathrm{CHA}_{2} \mathrm{DS}_{2}$-VASc Score } \\
\hline & $\mathrm{CHADS}_{2}$ Score & & \\
\hline CHF (recent) & 1 & $\begin{array}{l}\text { CHF, LV } \\
\text { Dysfunction }\end{array}$ & 1 \\
\hline $\begin{array}{l}\text { Hypertension } \\
\text { (history) }\end{array}$ & 1 & Hypertension & 1 \\
\hline Age $>75$ years & 1 & Diabetes & 1 \\
\hline Diabetes & 1 & $\begin{array}{l}\text { Vascular Disease } \\
\text { (Prior MI, PVD, } \\
\text { Aortic Plaque) }\end{array}$ & 1 \\
\hline Stroke/TIA & 2 & Female Gender & 1 \\
\hline \multirow[t]{4}{*}{ MAXIMUM SCORE } & 6 & Age $65-74$ years & 1 \\
\hline & & Age $>75$ years & 2 \\
\hline & & Stroke/TIA & 2 \\
\hline & & MAXIMUM SCORE & 9 \\
\hline
\end{tabular}

" Gage BF et al. JAMA. 2001;285:2864-70." Lip GYH et al. Chest 2010; 137: 263-72.

study by Coppens. ${ }^{[16]}$ Patients with a $\mathrm{CHADS}_{2}$ score of 0 had an overall annual stroke rate of $1.59 \%$. However, after reclassification of these patients with a $\mathrm{CHADS}_{2}$ score of 0 , the resulting $\mathrm{CHA}_{2} \mathrm{DS}_{2}$ VASc scores in these patients ranged from 0 to 3 . Based upon the reclassified $\mathrm{CHA}_{2} \mathrm{DS}_{2}$-VASc scores, the patients in this study had increasing annual relative stroke risks ranging from 0.84 for a $\mathrm{CHA}_{2} \mathrm{DS}_{2}-\mathrm{VAS}$ c score of $0,1.75$ for a $\mathrm{CHA}_{2} \mathrm{DS}_{2}-\mathrm{VAS}$ c score of 1 , 2.69 for a $\mathrm{CHA}_{2} \mathrm{DS}_{2}-\mathrm{VAS}$ c score of 2 and 3.20 for a score of 3 . In another study, $25 \%$ of the patients with a $\mathrm{CHADS}_{2}$ score of 0 were reclassified as having a $\mathrm{CHA}_{2} \mathrm{DS}_{2}-\mathrm{VASc}$ score of 0 or 1 and $43 \%$ were reclassified as having a $\mathrm{CHA}_{2} \mathrm{DS}_{2}-\mathrm{VASc}$ score of 0 to 2 . All of these patients with reclassified $\mathrm{CHA}_{2} \mathrm{DS}_{2}$-VASc scores of 0 to 2 had a lower thromboembolic event rate than did the patients in the original $\mathrm{CHADS}_{2}$ paper with a $\mathrm{CHADS}_{2}$ score of $0 .{ }^{[9],[10]} \mathrm{A}$ subsequent external validation analysis similarly compared the differential ability of the $\mathrm{CHADS}_{2}$ and $\mathrm{CHA}_{2} \mathrm{DS}_{2}$-VASc algorithms to stratify AF patients at risk for a number of clinical outcomes. In this analysis the $\mathrm{CHA}_{2} \mathrm{DS}_{2}-\mathrm{VASc}$ algorithm was better than the $\mathrm{CHADS}_{2}$-algorithm in predicting hospitalization due to thromboembolism, death due to thromboembolism and all cause mortality up to 10 years after the initial risk assessment. ${ }^{[11]}$

Accordingly, the available data suggest that the $\mathrm{CHA}_{2} \mathrm{DS}_{2}-\mathrm{VASc}$ algorithm is a more robust risk stratification tool. Based upon these and other comparative studies, the $\mathrm{CHA}_{2} \mathrm{DS}_{2}-\mathrm{VASc}$ score appears significantly more effective than the $\mathrm{CHADS}_{2}$ score in identifying truly low risk patients in whom oral anticoagulation can be avoided. Oral Anticoagulation Guideline Recommendations For Atrial Fibrillation Patients

The true benefit of a clinically-based algorithm stems from its ability to predict accurately clinical outcomes and secondarily, as a result of its predicative capability, its ability to drive clinical decision-making. Based upon a Markov assessment addressing the potential benefit of anticoagulant therapy among AF patients, Eckman suggested that, when the annual CVA risk is $<0.9 \%$, anticoagulant therapy is not indicated. ${ }^{[17]}$ In this analysis he proposed that the tipping point

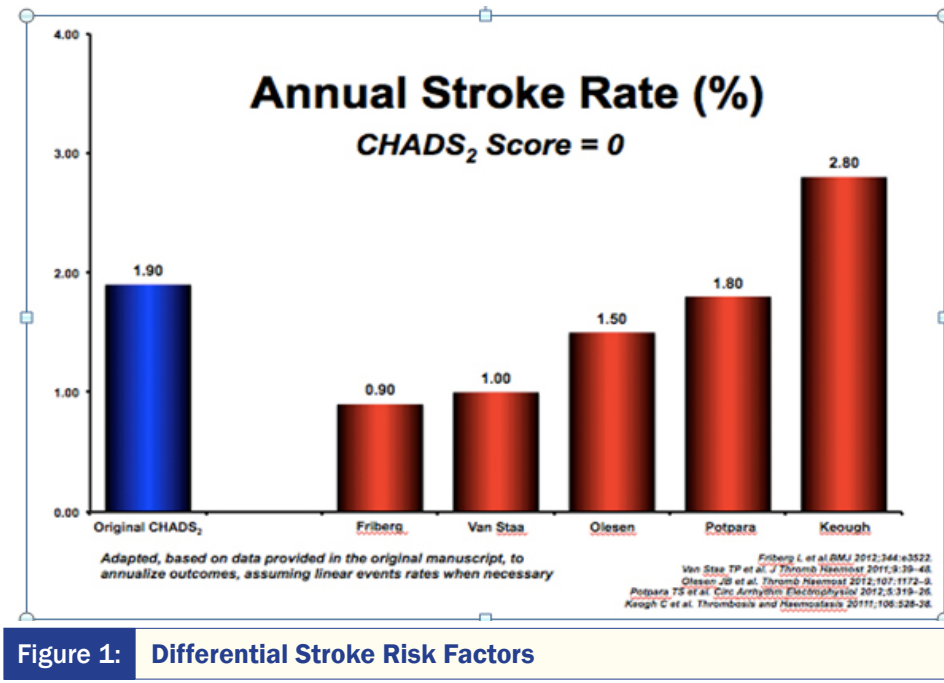

in favor of recommending chronic oral anticoagulation was found somewhere between an annual CVA risk of 0.9 to $1.7 \%$ based upon clinical features, the overall bleeding risk and the selected oral anticoagulant. Accordingly, given the annual stroke rates reported in the original $\mathrm{CHADS}_{2}$ paper, OAC would not be held in any patients. Based upon the annual event rates reported in the original $\mathrm{CHA}_{2} \mathrm{DS}_{2}$ VASc paper OAC would be held in all patients with a $\mathrm{CHA}_{2} \mathrm{DS}_{2}$ VASc score of 0 ; administered to all patients with a $\mathrm{CHA}_{2} \mathrm{DS}_{2}$ VASc score $\geq 2$, assuming that there was no contraindication, and of indeterminate benefit in patients with a $\mathrm{CHA}_{2} \mathrm{DS}_{2}-\mathrm{VASc}$ score of 1 .

On the basis of data derived from studies, assessing the ability of the $\mathrm{CHADS}_{2}$ and $\mathrm{CHA}_{2} \mathrm{DS}_{2}-\mathrm{VASc}$ scores to define thromboembolic risk, and statistical analyses, such as those performed by Eckberg, guidelines have been developed by the major cardiovascular and arrhythmia societies to assist clinicians in deciding when to use oral anticoagulant therapy in patients with AF. ${ }^{[18] .[19]}$ Accordingly, for patients with a $\mathrm{CHA}_{2} \mathrm{DS}_{2}-\mathrm{VASc}$ score of 0 the published ESC and AHA, ACC, HRS guidelines indicate that no anticoagulant therapy is warranted for patients with a $\mathrm{CHA}_{2} \mathrm{DS}_{2}-\mathrm{VASc}$ score of 0 while anticoagulation is recommended for patients with a $\mathrm{CHA}_{2} \mathrm{DS}_{2}$ VASc score $\geq 2$ in whom there are no contraindication. Both of these are class I indication recommendations. ${ }^{[18],[19]}$

Anticoagulation Controversies: Decision-Making In Atrial Fibrillation Patients With $\mathrm{A} \mathrm{CHA}_{2} \mathrm{DS}_{2}-\mathrm{VASc}$ Score Of 1

Significant controversy persists, however, upon what anticoagulant decisions constitute the best approach among patients with a $\mathrm{CHA}_{2} \mathrm{DS}_{2}-\mathrm{VASc}$ score of 1 . This is a very important question as the data suggest that between $9.7 \%$ and $17.6 \%$ of $\mathrm{AF}$ patients have a $\mathrm{CHA}_{2} \mathrm{DS}_{2}-\mathrm{VASc}$ score of $1 .^{[10],[12],[20],[21]}$ Furthermore, the thromboembolic event rates across published studies with a $\mathrm{CHA}_{2} \mathrm{DS}_{2}-\mathrm{VAS}$ c score of 1 are quite broad, ranging from $0.5 \%$ to $6.6 \% .^{[12],[22]-[27]}$

Based upon the variable thromboembolic event rates observed in patients with a $\mathrm{CHA}_{2} \mathrm{DS}_{2}-\mathrm{VASc}$ score of 1 , the established guidelines differ slightly in their recommendations for anticoagulant usage in this patient population. The AHA/ACC/HRS guidelines, published in 2014, state that either no anticoagulant therapy or oral anticoagulation treatment "may be considered" for AF patients with a $\mathrm{CHA}_{2} \mathrm{DS}_{2}-\mathrm{VASc}$ score of 1 . $^{[18]}$ The 2012 ESC guidelines suggest that 
anticoagulant usage in this population "should be considered" for AF patients with a $\mathrm{CHA}_{2} \mathrm{DS}_{2}-\mathrm{VASc}$ score of $1 .{ }^{[19]}$ This subtle variation in the published guidelines creates a therapeutic conundrum for clinicians when faced with making decisions about anticoagulation in these patients. Four possible options are presented below to help inform and assist clinicians when trying to make these difficult decisions.

The first suggested process focuses upon how the individual risk factors operative in the algorithms are used in clinical practice. Patients suffering from heart failure in the original $\mathrm{CHADS}_{2}$ manuscript had to have a history of a "recent heart failure exacerbation", not just a history of heart failure, to have it included as a risk factor. Hypertension was defined in the original $\mathrm{CHADS}_{2}$ paper to include patients with a "history of hypertension" rather than a specific BP value at the time of enrollment. ${ }^{[9]}$ In the original $\mathrm{CHA}_{2} \mathrm{DS}_{2}-\mathrm{VASc}$ manuscript the definitions for hypertension and heart failure were not clearly provided. Additionally the vascular disease risk factor was defined to include patients with a prior myocardial infarction, peripheral artery disease or aortic plaque. ${ }^{[10]}$

Table 2: Stroke Risk Stratification with the ATRIA Scoring Algorithm

\begin{tabular}{lll} 
Risk Factor & No Prior CVA & Prior CVA \\
Age & & \\
$>85$ years & 6 & 9 \\
$75-84$ & 5 & 7 \\
$65-74$ & 3 & 7 \\
$<65$ & 0 & 8 \\
Female Gender & 1 & 1 \\
Diabetes & 1 & 1 \\
Heart Failure & 1 & 1 \\
Hypertension & 1 & 1 \\
Proteinuria eGFR $<45$ & 1 & 1 \\
mL/min/1.73m ${ }^{2}$ or ESRD & 1 & 1 \\
MAXIMUM SCORE & 12 & 15 \\
\hline
\end{tabular}

Singer DE et al. J Am Heart Assoc 2013;2:e000250.

The recognized risk factors, which constitute the $\mathrm{CHADS}_{2}$ and $\mathrm{CHA}_{2} \mathrm{DS}_{2}$-VASc scores are not always employed in clinical practice today in the manner in which they were developed and originally used. ${ }^{[9],[10]}$ Decision-making in all clinical arenas frequently does morph over time as original criteria are often applied in a broader manner. Thus, advancements in diagnostic testing and indication creep' may lead to new ways in which these risk factors are defined and used that are very different from those observed in the original studies. Accordingly, the event rates observed among patients with particular risk factors might be very different today than they were when reported in those original studies because of an alteration in the employed definitions. For example, a patient with a positive calcium score, but no history of clinically active coronary disease, may have a very different stroke risk than a patient with a prior MI and significant peripheral vascular disease. Similarly a patient with recent heart failure secondary to a newly diagnosed tachycardia-mediated cardiomyopathy, which resolves with appropriate therapy, may not have the same thromboembolic risk as a patient with a long-standing cardiomyopathy associated with a chronically reduced ejection fraction but no recent heart failure.

This 'indication creep' places the decision-making processes into a more tenuous arena in which the scientific support in the
Table 3:

Bleeding Risk Stratification with the HAS-BLED Algorithm

Risk Factor

Score

Labile INR's

Bleeding Predisposition

Age $>65$ years or Frailty 1

Hypertension with SBP > $160 \mathrm{~mm} \mathrm{Hg}$

Prior CVA

Bleeding Risk Drugs or Alcohol Abuse

Abnormal Renal or Hepatic Function

MAXIMUM SCORE

Pisters R et al. Chest. 2010;138:1093-1100.

literature is often lacking. Therefore, when clinicians are faced with recommending treatment approaches to patients, a strict application of the original definitions is more likely to yield results in line with those reported by the authors in the original research papers. Thus, an important first step for clinicians, contemplating anticoagulation decisions among $\mathrm{AF}$ patients with a borderline $\mathrm{CHA}_{2} \mathrm{DS}_{2}$-VASc score, is to ensure that the manner in which the point assignments are created is in alignment with the original definitions as presented in the published literature. Acknowledging the limitations of the risk stratification algorithms and the guidelines upon which they were built can sometimes inform the decision-making process in a borderline patient.

Secondly, it is important to realize that each of the risk factors, which comprise the $\mathrm{CHADS}_{2}$ and the $\mathrm{CHA}_{2} \mathrm{DS}_{2}$-VASc scores, may not be equal in their predictive accuracy. For example, in the van Staa study, the thromboembolic risk associated with hypertension was proportional to the level of observed hypertension. ${ }^{[13]}$ The annual stroke risk increased proportionally from a baseline relative risk of 1 for a systolic $\mathrm{BP}$ of $120 \mathrm{~mm} \mathrm{HG}$ to a greater than 4-fold risk among patients with a systolic BP $>180 \mathrm{~mm}$ HG. Similarly in two other studies the thromboembolic risk among patients in whom female gender, heart failure, diabetes, vascular disease and hypertension constituted risk factors was similar. ${ }^{[1],[12]}$ However, in both of these studies those patients with a prior CVA/TIA or advanced age had a substantially higher stroke risk during follow-up, indicating that these two risk factors were associated with a higher risk than other risk factors [Figure 2]. In the Olesen study patients with combinations of risk factors had different risk profiles. For example patients with a $\mathrm{CHA}_{2} \mathrm{DS}_{2}-\mathrm{VASc}$ score of 2 in whom the two risk factors were female gender and heart failure had a relatively low risk with a hazard ratio for thromboembolic complications of 1.32 while patients with hypertension and heart failure had a substantially higher hazard ratio for thromboembolic complications of 4.19..$^{[11]}$

Accordingly, assessment of the particular risk factors present may result in allocating patients to higher or lower risk groups based upon the specific risk factors. For example, a 43-year-old male patient with mild, well-controlled diabetes may have a different thromboembolic risk than a 74 year old male patient without other risk factors even though they both have a $\mathrm{CHA}_{2} \mathrm{DS}_{2}-\mathrm{VASc}$ of 1 . Similarly an asymptomatic 48-year-old patient with minimal CAD detected on a CT angiogram may have a different risk than a patient of the same age with advanced coronary, peripheral vascular and cerebrovascular disease despite having the same $\mathrm{CHA}_{2} \mathrm{DS}_{2}$-VASc score. Therefore, differential assessment of the risk factors and judicious application thereof may be beneficial in borderline patients. 
A third consideration for clinicians, when trying to decide on whether to pursue oral anticoagulant therapy among AF patients with a $\mathrm{CHA}_{2} \mathrm{DS}_{2}-\mathrm{VASc}$ score of 1 , is to assess for the presence or absence of other risk factors potentially associated with an enhanced thromboembolic risk. Although the $\mathrm{CHADS}_{2}$ and $\mathrm{CHA}_{2} \mathrm{DS}_{2}-\mathrm{VASc}$ scores have become commonly used in the clinical sphere, based upon published guidelines, there is data suggesting that other clinical features, not included in these algorithms, can also help to clarify the thromboembolic risk. In some studies the presence of obstructive sleep apnea (OSA) in AF patients has been associated with a higher risk for an ischemic CVA. ${ }^{[28]}$ In addition, effective treatment for OSA in a number of studies has resulted in a reduction in that risk.

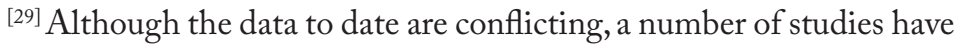
suggested a relationship between the degree of renal dysfunction and the thromboembolic risk in AF patients. ${ }^{[30],[31]}$ In the Loire Valley Atrial Fibrillation Project and the Tiawanese database PVD was associated with a higher thromboembolic risk among AF patients. [32],[33] Genetic and racial differences may also be associated with different thromboembolic risks. Several recent studies have noted a higher stroke risk among Asian and African-American patients with $\mathrm{AF}$ after adjusting for other risk factors. ${ }^{[33],[34]} \mathrm{A}$ number of anatomical considerations also may impact the stroke risk. For example, in one study, left atrial size correlated inversely with rates of stroke-free survival. ${ }^{[35]}$ In the SAVE and SCD-HeFT studies higher rates of stroke and thromboembolic event rates correlated with greater degrees of left ventricular systolic dysfunction. ${ }^{[36],[37]}$ Among patients with a borderline $\mathrm{CHA}_{2} \mathrm{DS}_{2}$-VASc score, assessing for the presence or absence of additional thromboembolic risk factors might prove advantageous.

Finally, although the $\mathrm{CHADS}_{2}$ and $\mathrm{CHA}_{2} \mathrm{DS}_{2}$-VASc scores have become a standard by which oral anticoagulant decisions are made, they are not the only algorithms available to assess risk. The ATRIA stroke risk score was developed by analyzing a population of patients participating in the Kaiser Permanante patient database [Table 2]. ${ }^{[38]}$ This score dichotomized patients into groups with and without a prior stroke then further analyzed patients based upon additional age and limited renal function breakouts while continuing to assess for the other characteristics included in the $\mathrm{CHADS}_{2}$ and $\mathrm{CHA}_{2} \mathrm{DS}_{2}-$ VASc scoring algorithms. Patients assessed with the ATRIA score have a total maximal potential score of 15 points. By using a larger number of operative clinical characteristics it was hoped that there

\begin{tabular}{ll|}
\hline Table 4: Bleeding Risk Stratification with the HEMORR ${ }_{2}$ HAGES Algorithm $^{-}$ & Score \\
Risk Factor & 1 \\
Hepatic or Renal Disease & 1 \\
Alcohol Abuse & 1 \\
Malignancy & 1 \\
Age > 75 years & 1 \\
Decreased Platelet Count or Function & 2 \\
Rebleeding Risk & 1 \\
Hypertension w/o Adequate Control & 1 \\
Anemia & 1 \\
Genetic Risk Factors & 1 \\
Elevated Falling Risk & 1 \\
Prior CVA & 12 \\
MAXIMUM ScoRE & \\
\hline
\end{tabular}

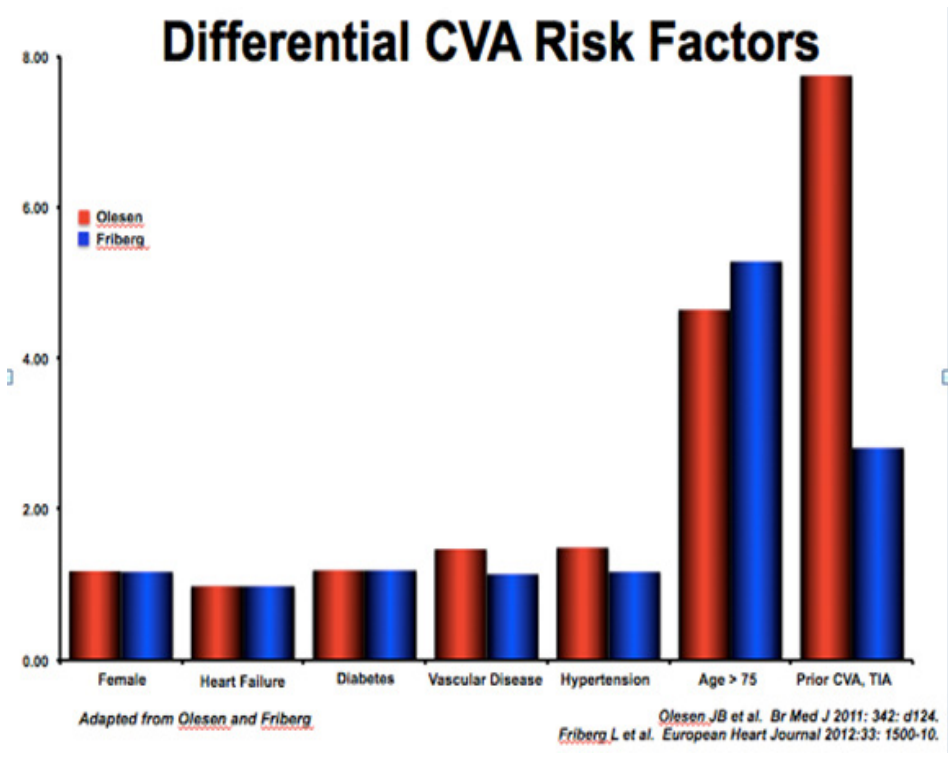

Figure 2: Variability of Stroke Risk among Patients with a $\mathrm{CHADS}_{2}$ Score of 0 might be greater risk differentiation possibilities.

When one compares risk stratification schemas, based on data presented in the original papers, $46.7 \%$ of the patients in the ATRIA manuscript were defined as low risk using the ATRIA stroke risk score (score of 0-5 points) vs. $49.7 \%$ of the patients in the CHADS manuscript in whom that risk was defined as low by the $\mathrm{CHADS}_{2}$ score (score of $0-1$ points) and only $7.6 \%$ of the patients in whom the risk was defined as low by the $\mathrm{CHA}_{2} \mathrm{DS}_{2}$-VASc score (score of 0 points) ${ }^{[9],[10],[38]}$ In each of these publications the annual stroke rates for these low risk groups were respectively $0.63 \%, 0.88 \%$ and $0.04 \%$. Although patients with a low $\mathrm{CHA}_{2} \mathrm{DS}_{2}$-VASc score, according to the original classification schema, have the lowest stroke event rate, unfortunately only $19.3 \%$ of the patients classified by the $\mathrm{CHA}_{2} \mathrm{DS}_{2}-$ VASc algorithm are characterized as low risk.

Therefore it appears difficult to identify a truly moderate sized low risk $\mathrm{CHA}_{2} \mathrm{DS}_{2}$-VASc patient group in whom the use of an OAC may not be needed. Presumably the patients within the 'high' risk $\mathrm{CHA}_{2} \mathrm{DS}_{2}$-VASc group ( $80.7 \%$ of the patients) have a variable risk, which might be better differentiated into low and high risk groups by using the ATRIA stroke risk score. When these groups were classified in Singer's study into low risk patients with an annual thromboembolic event rate per 100 person-years $<1 \%$ only $1.5 \%$ of these events were identified using the $\mathrm{CHADS}_{2}$ score, 9.9\% using the $\mathrm{CHA}_{2} \mathrm{DS}_{2}-\mathrm{VASc}$ score and $14.0 \%$ using the ATRIA stroke risk score. ${ }^{[38]}$ In a similar study, in which the ATRIA stroke risk algorithm was employed, $46.2 \%$ of the patients were characterized as low risk based upon an annual thromboembolic risk rate of $0.4-1.31 \% \cdot{ }^{[39]}$ The ATRIAL stroke risk score therefore may help to separate patients with a borderline $\mathrm{CHA}_{2} \mathrm{DS}_{2}$-VASc score into groups in whom OAC can be safely avoided and groups in whom treatment with an OAC would be clinically beneficial.

These findings beg the question about when and under what circumstances the ATRIA stroke risk score should be used. To this end the ATRIA stroke risk score appears most beneficial when applied to patients with a $\mathrm{CHA}_{2} \mathrm{DS}_{2}$-VASc score of 1 in whom there are concerns about serious bleeding to identify patients in whom the perceived thromboembolic risk seems to be very low so that one can avoid OAC therapy. Conversely, when the bleeding risk is felt to be 
acceptably low, the $\mathrm{CHA}_{2} \mathrm{DS}_{2}$-VASc score alone should be sufficient to determine the thromboembolic risk and dictate therapy. Caution should be exercised in using the ATRIA stroke risk score in isolation to avoid oral anticoagulation under-treatment.

In summary, when faced with providing patients with a borderline $\mathrm{CHA}_{2} \mathrm{DS}_{2}$-VASc of 1 with a recommendation to maximize clinical benefit, using the definitions in a strict and cautious manner; realizing that the individual risk criteria are different in their predictive capacity; considering other less well recognized clinical risk factors and using an alternative scoring tool as a supplement may allow one to reach a better clinical decision endpoint.

\section{The Role Of Bleeding Risk Assessment In Atrial Fibrillation}

\section{Patients}

Complete patient risk assessment also requires that one perform an analysis of the bleeding risk. A number of scoring tools (e.g. HAS BLED, ATRIA bleeding risk score, HEMORR ${ }_{2}$ HAGES, etc.) have been proposed to define the bleeding risk among AF patients [Table 3]-[Table 5]. ${ }^{[40]-[42]}$ The thromboembolic and bleeding risk factor algorithms overlap somewhat but to an incomplete degree. Unfortunately the predictive accuracy of all of these algorithms to identify patients at high risk for a major bleeding event is limited, as defined by the low c-statistic values. ${ }^{[43]}$ In direct comparisons and meta analyses the HAS-BLED score has had a better predictive accuracy than either the ATRIA Bleeding Risk Score or the HEMORR 2 HAGES score. ${ }^{[44],[45]}$ Accordingly HAS-BLED has become the standard algorithm to be used when attempting to define the bleeding risk in AF patients in whom oral anticoagulant therapy is being considered.

After determining the thromboembolic and bleeding risk rates one must then render a decision on whether to initiate or withhold anticoagulant therapy. Although bleeding is certainly a problematic clinical issue and major bleeding a serious clinical concern, ordinarily cerebral ischemic events, whether they are embolic, thrombotic or hemorrhagic, are frequently life-altering events with more negative clinical impact than most non-cerebral bleeding events. In one study among elderly AF patients at risk for both an embolic CVA and significant bleeding the quality adjusted life year benefit was significantly better among the patients treated with anticoagulation when compared to those in whom no anticoagulation was administered ${ }^{[46]}$ In the Swedish National Discharge Registry (HDR) Friberg demonstrated that the net clinical benefit favored oral anticoagulation in almost all $\mathrm{AF}$ patients except for those with a very low ischemic stroke risk (i.e. $\mathrm{CHA}_{2} \mathrm{DS}_{2}$-VASc score of 0 ) or a very high bleeding risk. ${ }^{[47]}$

Based upon the available information the default decision should be directed toward chronic anticoagulant therapy. Therefore, rather than precluding a decision to anticoagulate patients with an elevated

\section{Table 5: Bleeding Risk Stratification with the ATRIA Bleeding Algorithm}

\begin{tabular}{ll} 
Risk Factor & Score \\
Hypertension & 1 \\
Prior Hemorrhage & 1 \\
Age $>75$ years & 2 \\
Anemia & 3 \\
Significant Renal Disease (eGFR $<30 \mathrm{~mL} / \mathrm{min}$ & 3 \\
or Dialysis & 10 \\
MAXIMUM SCORE & \\
\hline Fang MC et al. J Am Coll Cardiol 2011:58:395-401.
\end{tabular}

$\mathrm{CHA}_{2} \mathrm{DS}_{2}$-VASc score, an elevated HAS-BLED score should lead to a focused approach directed at reducing the associated bleeding risk. Efforts to control blood pressure; limit alcohol use and provide for a stable environment in which the fall risk is minimized should be undertaken. A critical assessment of the potential benefit associated with concomitant usage of antiplatelet agents and non-steroidal anti-inflammatory agents should be performed. The existence of consistently elevated or labile INRs might lead one to recommend that a NOAC be used in place of warfarin. Accordingly, the bleeding risk scores, when elevated, should not be used to avoid anticoagulation, but alternatively, should be used to inform the management decisionmaking process toward a focus upon the modification of associated bleeding risk factors.

\section{The Global Atrial Fibrillation Oral Antricoagulation}

\section{Reportcard}

Given the overall benefit of chronic oral anticoagulant therapy among AF patients in preventing thromboembolic events, especially stroke, OAC should be standard practice in the clinical arena for patients with an appropriately defined risk in whom there are no contra-indications. Similarly the use of oral anticoagulants among patients with a low thromboembolic risk should not be undertaken in most clinical scenarios. Unfortunately the available data on oral anticoagulation usage rates and patterns among $\mathrm{AF}$ patients suggests that practice patterns do not align with these recommendations.

In a study performed among patients with a $\mathrm{CHADS}_{2}$ score $>1$, selected from the National Cardiovascular Data Registry PINNACLE program between July 2008 and December 2009, Chan observed an overall rate of anticoagulant usage of only $55 \%$ with a range from approximately $25 \%$ to $~ 80 \% .48$ In another study, evaluating patients in the UK with a history of AF, $39.7 \%$ of patients with a $\mathrm{CHA}_{2} \mathrm{DS}_{2}-$ VASc score $>2$ and $39.5 \%$ of patients with a $\mathrm{CHADS}_{2}$ score $>1$ were not receiving appropriate OAC therapy. During a 12 -month follow-up, anticoagulated patients had statistically lower CVA (OR: 0.60, CI: 0.45-0.81) and death (OR: 0.54, CI: 0.38-0.75) rates, $\mathrm{p}<$ $0.001 .{ }^{[49]}$

Among patients enrolled in the large international "Global Anticoagulant Registry in the FIELD (GARFIELD)" registry, guideline-driven oral anticoagulation decisions did not appear to be commonplace. ${ }^{[50]}$ In patients with $\mathrm{CHADS}_{2}$ and $\mathrm{CHA}_{2} \mathrm{DS}_{2}$-VASc scores $>2$ only $62.0 \%$ and $59.3 \%$ of patients respectively received oral anticoagulant therapy. Unfortunately many low risk patients (i.e. $42.5 \%$ of patients with a $\mathrm{CHADS}_{2}$ score of 0 and $38.7 \%$ of patients with a $\mathrm{CHA}_{2} \mathrm{DS}_{2}$-VASc score of 0 ) were administered oral anticoagulant therapy, exposing them to a bleeding risk not in general justified by the presently available data. KAKKA

Inappropriate oral anticoagulation therapy in $\mathrm{AF}$ patients, when not indicated because of a low thromboembolic risk, and the failure to initiate anticoagulant therapy in at risk AF patients, when indicated by established guidelines, both constitute a failure to deliver care according to defined quality directives. Clinicians individually and organizations systemically should work together to put into place processes, which ensure the initiation and maintenance of therapeutic approaches that align with established standards of care. By doing such we will improve the likelihood of advancing better clinical outcomes.

Summary

Atrial fibrillation is a common medical problem, which is expected 
to increase globally both in incidence and prevalence, given an aging population that has a higher prevalence of clinical problems associated with its development. Stroke is an unfortunate and perhaps the most devastating complication observed among patients with AF. Effective thromboembolic risk stratification and the employment of therapeutic interventions to minimize that risk in patients at risk can result in substantial health and socio-economic benefits.

For most patients the $\mathrm{CHA}_{2} \mathrm{DS}_{2}-\mathrm{VASc}$ score permits clinicians to define effectively the thromboembolic risk. The available data suggests that it is better than the CHADS 2 score, especially when trying to identify low risk patients. For patients on either side of the risk-benefit spectrum with either a $\mathrm{CHA}_{2} \mathrm{DS}_{2}-\mathrm{VASc}$ score of 0 or > 2 the therapeutic decision-making process is usually rather simple in the absence of anticoagulation contraindications.

However, therapeutic controversies concerning long-term oral anticoagulation therapy exist. For borderline patients with a $\mathrm{CHA}_{2} \mathrm{DS}_{2}$-VASc score of 1 additional considerations (e.g. analyzing the particular components of the individual risk factors, weighing the individual thromboembolic event rates associated with each of the defined risk factors, including additional risk factors in the assessment equation or using a supplemental risk sore algorithm [e.g. the ATRIA stroke risk score]) may result in a more informed and hopefully better clinical decision.

Similarly, for patients with a high bleeding risk the performance of a bleeding assessment enhances the decision-making process. For most AF patients, with a $\mathrm{CHA}_{2} \mathrm{DS}_{2}$-VASc score for whom oral anticoagulation is recommended, the risk: benefit analysis will favor the employment of oral anticoagulation. For many if not most of those patients with an augmented thromboembolic and bleeding risk, careful consideration and modification of an associated lifestyle activity and/or alternative pharmacological anticoagulation options is a requisite part of the patient engagement process. In a small number of at risk patients the risk: benefit analysis will result in a clinical decision to avoid oral anticoagulation.

Perhaps the most important component necessary to facilitate addressing effectively this issue at the individual and societal level is the realization that existing scenarios in place to render clinical decisions have failed to establish a high degree of adherence to established practice guidelines. When practicing in situations without established and effective processes, clinicians often fail to provide best-in-breed thromboembolic risk decisions to their patients. In some cases the decisions create unnecessary bleeding risk when oral anticoagulants are inappropriately recommended. In other scenarios they fail to mitigate the thromboembolic risk when oral anticoagulants are not given to patients in whom the guidelines recommend that they be administered. Accordingly, clinicians and healthcare organizations should hold themselves individually and collectively responsible to ensure better clinical outcomes. They should work together to establish, within their institutions, systemic processes, which will augment the likelihood that excellent clinical outcomes will manifest. By addressing these issues at the individual and societal level we adhere to the ethical tenets of our profession; improve the quality of life as experienced by our patients and advance the overall health of our regional and global communities.

\section{Conflict Of Interests}

None.

\section{Disclosures}

None.

\section{References}

1. Naccarelli Gerald V, VarkerHelen, LinJay, SchulmanKathy L. Increasing prevalence of atrial fibrillation and flutter in the United States. Am. J. Cardiol. 2009;104 (11):1534-9.

2. Stefansdottir Hrafnhildur, AspelundThor, GudnasonVilmundur, ArnarDavid O. Trends in the incidence and prevalence of atrial fibrillation in Iceland and future projections. Europace. 2011;13 (8):1110-7.

3. http://www.worldlifeexpectancy.com/cause-ofdeath/stroke/by-country/.. 0;0:0-0.

4. O’Donnell Martin J, XavierDenis, LiuLisheng, ZhangHongye, ChinSiu Lim, Rao-MelaciniPurnima, RangarajanSumathy, IslamShofiqul, PaisPrem, McQueenMatthew J, MondoCharles, DamascenoAlbertino, LopezJaramilloPatricio, HankeyGraeme J, DansAntonio L, YusoffKhalid, TruelsenThomas, DienerHans-Christoph, SaccoRalph L, RyglewiczDanuta, CzlonkowskaAnna, WeimarChristian, WangXingyu, YusufSalim. Risk factors for ischaemic and intracerebral haemorrhagic stroke in 22 countries (the INTERSTROKE study): a case-control study. Lancet. 2010;376 (9735):112-23.

5. Kimura K, MinematsuK, YamaguchiT. Atrial fibrillation as a predictive factor for severe stroke and early death in 15,831 patients with acute ischaemic stroke. J. Neurol. Neurosurg. Psychiatr. 2005;76 (5):679-83.

6. Sussman Matthew, MenzinJoseph, LinIris, KwongWinghan J, MunsellMichael, FriedmanMark, SelimMagdy. Impact of atrial fibrillation on stroke-related healthcare costs. J Am Heart Assoc. 2013;2 (6).

7. Campbell HA et al. Journal of Biological Chemistry. 1940;138:21-23.

8. Hart R G, BenaventeO, McBrideR, PearceL A. Antithrombotic therapy to prevent stroke in patients with atrial fibrillation: a meta-analysis. Ann. Intern. Med. 1999;131 (7):492-501.

9. Gage B F, WatermanA D, ShannonW, BoechlerM, RichM W, RadfordM J. Validation of clinical classification schemes for predicting stroke: results from the National Registry of Atrial Fibrillation. JAMA. 2001;285 (22):2864-70.

10. Lip Gregory Y H, NieuwlaatRobby, PistersRon, LaneDeirdre A, CrijnsHarry J G $M$. Refining clinical risk stratification for predicting stroke and thromboembolism in atrial fibrillation using a novel risk factor-based approach: the euro heart survey on atrial fibrillation. Chest. 2010;137 (2):263-72.

11. Olesen Jonas Bjerring, LipGregory Y H, HansenMorten Lock, HansenPeter Riis, TolstrupJanne Schurmann, LindhardsenJesper, SelmerChristian, AhlehoffOle, OlsenAnne-Marie Schjerning, GislasonGunnar Hilmar, TorpPedersenChristian. Validation of risk stratification schemes for predicting stroke and thromboembolism in patients with atrial fibrillation: nationwide cohort study. BMJ. 2011;342.

12. Friberg Leif, RosenqvistMårten, LipGregory Y H. Evaluation of risk stratification schemes for ischaemic stroke and bleeding in 182678 patients with atrial fibrillation: the Swedish Atrial Fibrillation cohort study. Eur. Heart J. 2012;33 (12):1500-10.

13. Van Staa T P, SetakisE, Di TannaG L, LaneD A, LipG Y H. A comparison of risk stratification schemes for stroke in 79,884 atrial fibrillation patients in general practice. J. Thromb. Haemost. 2011;9 (1):39-48.

14. Potpara Tatjana S, PolovinaMarija M, LicinaMarina M, MarinkovicJelena M, ProstranMilica S, LipGregory Y H. Reliable identification of "truly low" thromboembolic risk in patients initially diagnosed with "lone" atrial fibrillation: the Belgrade atrial fibrillation study. Circ Arrhythm Electrophysiol. 2012;5 (2):319-26.

15. Keogh Claire, WallaceEmma, DillonCiara, DimitrovBorislav D, FaheyTom. Validation of the CHADS2 clinical prediction rule to predict ischaemic stroke. A systematic review and meta-analysis. Thromb. Haemost. 2011;106 (3):528-38.

16. Coppens M et al. Throm Haemost. 2012;107:1172-1179.

17. Eckman Mark H, SingerDaniel E, RosandJonathan, GreenbergSteven M. Moving the tipping point: the decision to anticoagulate patients with atrial fibrillation. 
Circ Cardiovasc Qual Outcomes. 2011;4 (1):14-21.

18. January CT et al. J Am Coll Cardiol. 2014;64:2305-2307.

19. Camm A John, LipGregory Y H, De CaterinaRaffaele, SavelievaIrene, AtarDan, HohnloserStefan H, HindricksGerhard, KirchhofPaulus. 2012 focused update of the ESC Guidelines for the management of atrial fibrillation: an update of the 2010 ESC Guidelines for the management of atrial fibrillation. Developed with the special contribution of the European Heart Rhythm Association. Eur. Heart J. 2012;33 (21):2719-47.

20. Kirchhof Paulus, LipGregory Y H, Van GelderIsabelle C, BaxJeroen, HylekElaine, KääbStefan, SchottenUlrich, WegscheiderKarl, BorianiGiuseppe, EzekowitzMichael, DienerHans, HeidbuchelHein, LaneDeirdre, MontLuis, WillemsStephan, DorianPaul, VardasPanos, BreithardtGünter, CammA John. Comprehensive risk reduction in patients with atrial fibrillation: Emerging diagnostic and therapeutic options. Executive summary of the report from the 3rd AFNET/EHRA consensus conference. Thromb. Haemost. 2011;106 (6):1012-9.

21. ChaoTze-Fan,LinYenn-Jiang,TsaoHsuan-Ming,TsaiChin-Feng,LinWei-Shiang, ChangShih-Lin, LoLi-Wei, HuYu-Feng, TuanTa-Chuan, SuenariKazuyoshi, LiCheng-Hung, HartonoBeny, ChangHung-Yu, AmbroseKibos, WuTsu-Juey, ChenShih-Ann. CHADS(2) and CHA(2)DS(2)-VASc scores in the prediction of clinical outcomes in patients with atrial fibrillation after catheter ablation. J. Am. Coll. Cardiol. 2011;58 (23):2380-5.

22. Friberg Leif, SkeppholmMika, TeréntAndreas. Benefit of anticoagulation unlikely in patients with atrial fibrillation and a CHA2DS2-VASc score of 1. J. Am. Coll. Cardiol. 2015;65 (3):225-32.

23. Komatsu Takashi, SatoYoshihiro, OzawaMahito, KunugitaFusanori, YoshizawaReisuke, MorinoYoshihiro, NakamuraMotoyuki. Comparison between CHADS2 and CHA2DS2-VASc score for risk stratification of ischemic stroke in Japanese patients with non-valvular paroxysmal atrial fibrillation not receiving anticoagulant therapy. Int Heart J. 2014;55 (2):119-25.

24. Lip Gregory Y H, LarocheCécile, PopescuMircea Iaochim, RasmussenLars Hvilsted, Vitali-SerdozLaura, DanGheorghe-Andrei, KalarusZbigniew, CrijnsHarry J G M, OliveiraMario Martins, TavazziLuigi, MaggioniAldo P, BorianiGiuseppe. Improved outcomes with European Society of Cardiology guideline-adherent antithrombotic treatment in high-risk patients with atrial fibrillation: a report from the EORP-AF General Pilot Registry. Europace. 2015;17 (12):1777-86

25. Chao Tze-Fan, LiuChia-Jen, WangKang-Ling, LinYenn-Jiang, ChangShih-Lin, LoLi-Wei, HuYu-Feng, TuanTa-Chuan, ChungFa-Po, LiaoJo-Nan, ChenTzengJi, LipGregory Y H, ChenShih-Ann. Incidence and prediction of ischemic stroke among atrial fibrillation patients with end-stage renal disease requiring dialysis. Heart Rhythm. 2014;11 (10):1752-9.

26. Huang Duo, AnguoLuo, YueWen-Sheng, YinLixue, TseHung-Fat, SiuChungWah. Refinement of ischemic stroke risk in patients with atrial fibrillation and CHA2 DS2 -VASc score of 1. Pacing Clin Electrophysiol. 2014;37 (11):1442-7.

27. Siu Chung-Wah, LipGregory Y H, LamKwok-Fai, TseHung-Fat. Risk of stroke and intracranial hemorrhage in 9727 Chinese with atrial fibrillation in Hong Kong. Heart Rhythm. 2014;11 (8):1401-8.

28. Lipford Melissa C, FlemmingKelly D, CalvinAndrew D, MandrekarJay, BrownRobert D, SomersVirend K, CaplesSean M. Associations between Cardioembolic Stroke and Obstructive Sleep Apnea. Sleep. 2015;38 (11):1699_ 705.

29. Yaranov Dmitry M, SmyrlisAthanasios, UsatiiNatalia, ButlerAmber, PetriniJoann R, MendezJose, WarshofskyMark K. Effect of obstructive sleep apnea on frequency of stroke in patients with atrial fibrillation. Am. J. Cardiol. 2015;115 (4):461-5.

30. Go Alan S, FangMargaret C, UdaltsovaNatalia, ChangYuchiao, PomernackiNiela K, BorowskyLeila, SingerDaniel E. Impact of proteinuria and glomerular filtration rate on risk of thromboembolism in atrial fibrillation: the anticoagulation and risk factors in atrial fibrillation (ATRIA) study. Circulation. 2009;119 (10):1363-9.
31. Zeng Wu-Tao, SunXiu-Ting, TangKai, MeiWei-Yi, LiuLi-Juan, XuQing, ChengYun-Jiu. Risk of thromboembolic events in atrial fibrillation with chronic kidney disease. Stroke. 2015;46 (1):157-63.

32. Olesen JB et al. Chest. 2012;141:147-153.

33. Lin Lian-Yu, LeeChang-Hsing, YuChih-Chieh, TsaiChia-Ti, LaiLing-Pin, HwangJuey-Jen, ChenPau-Chung, LinJiunn-Lee. Risk factors and incidence of ischemic stroke in Taiwanese with nonvalvular atrial fibrillation-- a nation wide database analysis. Atherosclerosis. 2011;217 (1):292-5.

34. Kabra Rajesh, GirotraSaket, Vaughan SarrazinMary. Refining Stroke Prediction in Atrial Fibrillation Patients by Addition of African-American Ethnicity to CHA2DS2-VASc Score. J. Am. Coll. Cardiol. 2016;68 (5):461-70.

35. Bouzas-Mosquera Alberto, BroullónFrancisco J, Álvarez-GarcíaNemesio, MéndezElizabet, PeteiroJesús, Gándara-SambadeTeresa, PradaOscar, MosqueraVíctor X, Castro-BeirasAlfonso. Left atrial size and risk for all-cause mortality and ischemic stroke. CMAJ. 2011;183 (10):E657-64.

36. Loh E, SuttonM S, WunC C, RouleauJ L, FlakerG C, GottliebS S, LamasG A, MoyéL A, GoldhaberS Z, PfefferM A. Ventricular dysfunction and the risk of stroke after myocardial infarction. N. Engl. J. Med. 1997;336 (4):251-7.

37. Freudenberger Ronald S, HellkampAnne S, HalperinJonathan L, PooleJeanne, AndersonJill, JohnsonGeorge, MarkDaniel B, LeeKerry L, BardyGust H. Risk of thromboembolism in heart failure: an analysis from the Sudden Cardiac Death in Heart Failure Trial (SCD-HeFT). Circulation. 2007;115 (20):2637-41.

38. Singer Daniel E, ChangYuchiao, BorowskyLeila H, FangMargaret C, PomernackiNiela K, UdaltsovaNatalia, ReynoldsKristi, GoAlan S. A new risk scheme to predict ischemic stroke and other thromboembolism in atrial fibrillation: the ATRIA study stroke risk score. J Am Heart Assoc. 2013;2 (3).

39. van den Ham Hendrika A, KlungelOlaf H, SingerDaniel E, LeufkensHubert G M, van StaaTjeerd P. Comparative Performance of ATRIA, CHADS2, and CHA2DS2-VASc Risk Scores Predicting Stroke in Patients With Atrial Fibrillation: Results From a National Primary Care Database.J. Am. Coll. Cardiol. 2015;66 (17):1851-9.

40. Pisters Ron, LaneDeirdre A, NieuwlaatRobby, de VosCees B, CrijnsHarry J G M, LipGregory Y H. A novel user-friendly score (HAS-BLED) to assess 1-year risk of major bleeding in patients with atrial fibrillation: the Euro Heart Survey. Chest. 2010;138 (5):1093-100.

41. Fang Margaret C, GoAlan S, ChangYuchiao, BorowskyLeila H, PomernackiNiela K, UdaltsovaNatalia, SingerDaniel E. A new risk scheme to predict warfarinassociated hemorrhage: The ATRIA (Anticoagulation and Risk Factors in Atrial Fibrillation) Study. J. Am. Coll. Cardiol. 2011;58 (4):395-401.

42. Gage Brian F, YanYan, MilliganPaul E, WatermanAmy D, CulverhouseRobert, RichMichael W, RadfordMartha J. Clinical classification schemes for predicting hemorrhage: results from the National Registry of Atrial Fibrillation (NRAF). Am. Heart J. 2006;151 (3):713-9.

43. Zhu Wengen, HeWenfeng, GuoLinjuan, WangXixing, HongKui. The HASBLED Score for Predicting Major Bleeding Risk in Anticoagulated Patients With Atrial Fibrillation: A Systematic Review and Meta-analysis. Clin Cardiol. 2015;38 (9):555-61.

44. Apostolakis Stavros, LaneDeirdre A, GuoYutao, BullerHarry, LipGregory Y H. Performance of the HEMORR(2)HAGES, ATRIA, and HAS-BLED bleeding risk-prediction scores in patients with atrial fibrillation undergoing anticoagulation: the AMADEUS (evaluating the use of SR34006 compared to warfarin or acenocoumarol in patients with atrial fibrillation) study. J. Am. Coll. Cardiol. 2012;60 (9):861-7.

45. Caldeira Daniel, CostaJoão, FernandesRicardo M, PintoFausto J, FerreiraJoaquim J. Performance of the HAS-BLED high bleeding-risk category, compared to ATRIA and HEMORR2HAGES in patients with atrial fibrillation: a systematic review and meta-analysis. J Interv Card Electrophysiol. 2014;40 (3):277-84.

46. Man-Son-Hing M, NicholG, LauA, LaupacisA. Choosing antithrombotic 
therapy for elderly patients with atrial fibrillation who are at risk for falls. Arch. Intern. Med. 1999;159 (7):677-85.

47. Friberg Leif, RosenqvistMårten, LipGregory Y H. Net clinical benefit of warfarin in patients with atrial fibrillation: a report from the Swedish atrial fibrillation cohort study. Circulation. 2012;125 (19):2298-307.

48. Chan Paul S, MaddoxThomas M, TangFengming, SpinlerSarah, SpertusJohn A. Practice-level variation in warfarin use among outpatients with atrial fibrillation (from the NCDR PINNACLE program). Am. J. Cardiol. 2011;108 (8):1136-40.

49. Shantsila Eduard, WolffAndreas, LipGregory Y H, LaneDeirdre A. Optimising stroke prevention in patients with atrial fibrillation: application of the GRASP-AF audit tool in a UK general practice cohort. Br J Gen Pract. 2015;65 (630):e16-23.

50. Kakkar AK et al. PLoS One. 2013;8:634-679. 